\section{A fatal case of pulmonary anthrax}

Pulmonary anthrax is rare in Great Britain. This case illustrates some diagnostic problems and the possibility of infection from horticultural bone meal fertiliser.

\section{Case report}

A 53-year-old clerk in an engineering firm was seen initially with a threeday history of sore throat, pyrexia, chest pains, and slight abdominal tenderness. He was treated with amoxycillin and diazepam and referred to the casualty department, but discharged home when no other physical signs were detected. That night he developed worsening chest and abdominal pains, dyspnoea, and a cough. He was readmitted to hospital, where he died one hour later. On admission he was disorientated, pyrexial $\left(38.5^{\circ} \mathrm{C}\right)$ severely dyspnoeic, and cyanosed. He had a tachycardia (120 per minute) constricted pupils, neck rigidity, and a tense abdomen. Blood examination showed haemoconcentration (haemoglobin $21.3 \mathrm{~g} / \mathrm{dl}$, packed cell volume $61 \cdot 1 \%$, and white blood count $19 \times 10^{9} / 1\left(19000 / \mathrm{mm}^{3}\right)$ ) and acidosis Treatment with $8.4 \%$ sodium bicarbonate intraveneously, isoprenaline, hydrocortisone, and calcium gluconate was not beneficial.

A chest $x$-ray examination showed consolidation of the right mid-zone and lower zone and right pleural effusion and broadening of the superior mediastinal shadow (see figure). Blood cultures yielded Bacillus anthracis which showed resistance to pencillin (MIC $>10 \mathrm{mg} / \mathrm{l}$ ) but sensitivity to tetracycline, erythromycin, and gentamicin.

At necropsy there was a haemothorax and slightly collapsed and haemorrhagic right lung, with a $6-\mathrm{cm}$ diameter mass in the right anterior mediastinum. There was a large blood-stained ascites and gelatinous oedema of the serosa. The small intestines showed areas of congestion and ulceration with general mucosal inflammation. No: abdominal lymphadenopathy was present. The meninges were haemorrhagic over the superior surfaces of the cerebral hemispheres, and histologically showed early meningitic changes and numerous Gram-positive bacilli. Sections of the mediastinal mass and lymph nodes showed extensive necrotic and haemorrhagic changes with a proliferation of reactive primitive cells of lymphoid or histiocytic type, and microcolonies of organisms morphologically resembling Bacillus anthracis. Gram-positive bacilli resembling Bacillus anthracis were present in the ulcerated mucosal areas of the ileum. Apart from areas of congestion and the presence of few atypical lymphoid cells in the spleen, no other significant histological features were present in other organs.

Epidemiological investigations revealed no potential sources of anthrax except bone meal, which the patient had used in large quantities in his garden, while continuing to smoke. Bacteriological examination of remnants, other retailer samples, and random garden soil samples proved negative.

\section{Discussion}

The rapid progression of the illness made clinical and radiological diagnosis impossible, and the necropsy appearances difficult, to interpret

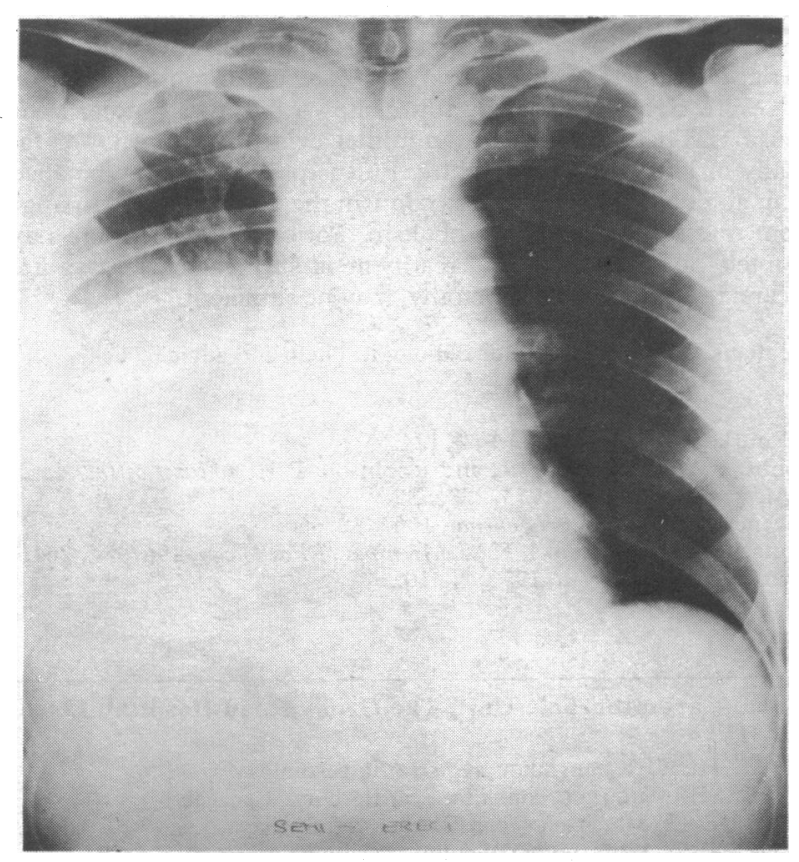

Chest $x$-ray film showing consolidation of right middle and lower zones. There is a right pleural effusion and broadening of the superior mediastinal shadow. initially without the benefit of a clinical or bacteriological diagnosis. The radiological appearances are identical to those described by others $^{1}$ and these may arouse suspicion. The histopathology of the mediastinal mass simulated a lymphoid neoplastic tumour but the recognition of microcolonies within the nodes suggested a reactive process and indicated inhalation as the route of infection.

The laboratory recognition of Bacillus anthracis may not be easy but characteristic morphology, production of capsules on $0.7 \%$ bicarbonate medium, and susceptibility to specific gammabacteriophage are diagnostic. Pencillin resistance is rare, although one case has been noted, ${ }^{2}$ and this must be considered when the response to pencillin treatment in anthrax is not prompt.

Probably in this case contaminated bone meal was the most likely source of the infecting organism.

I am grateful to Dr D. H G MacQuaide for permission to publish details of this case; to Dr R A Sladden for descriptions of the post-mortem and histological findings; to Dr J R Davies for carrying out specific gammabacteriophage tests on the strain isolated; and to $\mathrm{H}$ M Coroner for Northamptonshire for permission to report this case.

${ }^{1}$ Marc Laforce, F, et al, Archives of Environmental Health, 1969, 18, 798. 2 Christie, A B, Infectious Diseases, Epidemiology and Clinical Practice, 1st edn, p 771. Edinburgh, Livingstone, 1969.

Department of Pathology, General Hospital, Northampton M SEVERN, MB, MRCPATH, consultant microbiologist

\section{Crohn's disease of colon presenting as irritable bowel syndrome}

Crohn's disease of the colon may be present when sigmoidoscopy and radiography of the whole alimentary tract show no abnormality. Three such cases were noted during a survey of patients with Crohn's colitis and they serve to emphasise the importance of routine rectal biopsy when investigating cases of "irritable bowel syndrome."

\section{Case reports}

A man aged 30 gave a 10-year history of intermittent abdominal pain and diarrhoea. His stools were semi-formed and did not contain blood. Three barium meals and one barium enema had been performed during the previous three years. None had shown any abnormality. On examination he was tense and introspective. Sigmoidoscopy showed no abnormality and a diagnosis of irritable bowel syndrome was made. A barium enema and barium meal and follow-through showed no abnormality. A colonic motility study elicited enhanced responses to both food and neostigmine, consistent with the irritable bowel syndrome. A rectal biopsy specimen taken routinely at sigmoidoscopy, however, showed the typical sarcoid-type granulomata of Crohn's colitis (see fig)

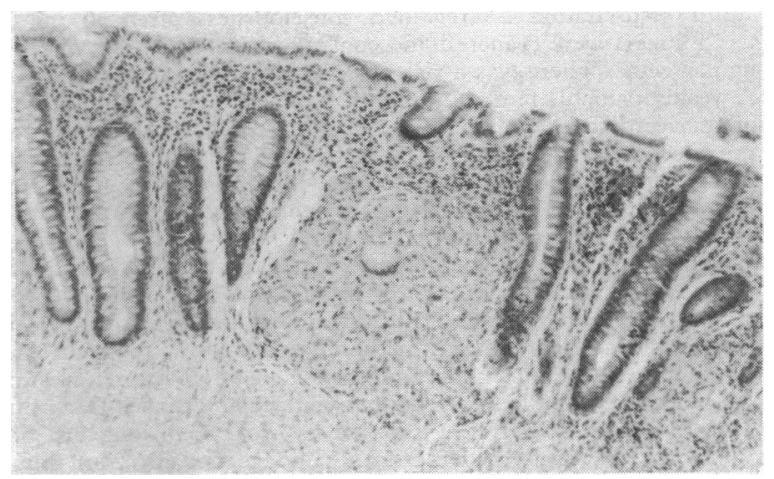

Photomicrograph of rectal biopsy in case $1(\times 5)$. 